\title{
Les Yeux d'Emma: Ensemble fendu ou indéchiffrable énigme?²
}

Margot MILLER

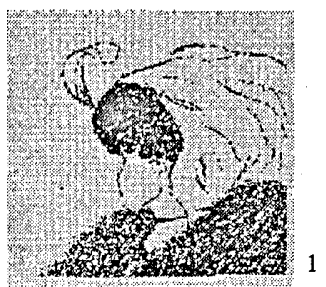

$\mathrm{C}$ omment aborder les femmes monstres ?Dans "Les yeux d'Emma," une nouvelle d'Antoine Chalvin, la référence aux "Yeux d'Elsa" de Louis Aragon saute immédiatement aux yeux et propose ainsi deux thèmes: d'un côté la dualité de la tragédie et de l'autre l'espoir et la résistance (Adereth 17). Le concept de la dualité seule ne suffit pas à l'analyser ce texte mais nous donne une première idée. Mettons-la de côté et lisons le récit. Deux femmes dissimulées sous des cagoules noires, font penser à "La femme cachée" de Colette, car ici aussi, le narrateur du récit ne comprend pas la femme. Au-delà de la dualité pensons alors à la dissimulation de la femme ou plutôt à l'impossibilité chez l'homme de la regarder et de la comprendre, comme dans les mythes de la Méduse ou d'Orphée. Mais comme il s'agit de deux femmes dans cette nouvelle, et surtout avec le dénouement "monstrueux," nous devons chercher plus loin encore.

Les têtes et les corps mélangés, que nous découvrons à la fin du récit, qui sont impossible à voir en même temps, font penser à l'image célèbre (ci-dessus), "Ma femme et ma belle-mère" (Block). Elle offre le portrait de deux femmes mais on ne semble pas pouvoir en voir qu' une des deux à la fois. Car l'esprit organise la figure contre l'arrière plan et lorsqu'on fait surgir l'une, l'autre disparaît. Cette image illustre le principe 
de la perception selon la théorie "Gestalt." ${ }^{3}$ Ce principe est tel que les parties prennent leur sens à partir des ensembles auxquelles elles appartiennent et ne peuvent être appréhendées hors de cette intégration (Wolman 159). Par ailleurs, le nom "Emma" signifie "complet," un tout, l'ensemble (Samuelson 73), tandis que les yeux symbolisent à la fois la perception intellectuelle (Chevalier 686) et l'âme de la personne, "l'un [des yeux] regarde le temps [qui fuit], l'autre est tourné vers l'éternité," (687). Voilà une piste intéressante que nous allons suivre, car le double portrait d'une vieille, la tête couverte par une cagoule, les yeux baissés qui regard peut-être la fuite du temps, et une belle jeune femme qui regarde ailleurs, et dont le foulard qui la coiffe semble flotter, suggère que la nouvelle de Chalvin peut être lue à travers cette image, à travers l'impossibilité (ou le refus?) de mettre ensemble l'image de l'une et de l'autre en même temps.

Le regard du lecteur est impliqué dans le regard de l'image, et de même dans le regard du narrateur qui est aussi celui qui subit l'expérience mystérieuse, voire monstrueuse, de trouver la Femme en deux personnes, puis de fendre les deux corps pour ainsi reconstruire un corps censé être "parfait" mais qui détruit en fin de compte l'image idéale, celle de la photographie trouvée dans la maison confondue avec celle que porte le narrateur que nous pouvons supposer être de sa femme. Regardons le récit de plus près.

Un jour, lors d'une randonnée de deux semaines, et sentant le poids de son sac à dos, un homme se retrouve fatigué, ayant soif et n'ayant plus rien à boire, dans la lande déserte. Soudain, une pauvre maison délabrée surgit devant lui (je souligne). Déjà nous sommes dans un milieu de mystère car les maisons se voient normalement de loin et ne surgissent pas. L'homme donne un coup d'œil par la fenêtre, ne voyant qu'une photographie d'une jeune et belle femme sur la cheminée. "Trois coups espacés suffirent à éveiller quelque chose à l'intérieur." "Quelque chose" (je souligne) nous signale encore le mystère, que nous avons peut-être affaire à une ou des non-personnes, des monstres peut-être? Donc il est intéressé par, mais en même temps inquiet face au lieu où semble habiter une belle jeune femme. À l'intérieur en fait habitent deux femmes dont l'une est vieille et l'autre jeune et, apparemment, belle. La vieille a le corps un peu voûté (88) et la jeune est grande, porte une robe blanche qui frissonne au vent (91); elle a des yeux verts, "presque fluorescents" (91). Le narrateur est fasciné par cette "perfection végétale." La couleur vert signifie "l'éveil des eaux primordiales [. . . éveil de la vie" (Chevalier 1002), donc les pluies du printemps qui entraînent la verdure et la vie. Le 
vert est le végétal, la reprise de vie que nous prenons pour la jeunesse, sous forme de deux métaphores mortes, LA VIE EST UNEANNÉE et LES PERSONNES SONT DES PLANTES. ${ }^{4}$ Nous savons maintenant que la jeune femme appelée Emma représente la vie, la jeunesse, la perfection, donc un idéal selon la conscience du narrateur.

La vieille lui donne à boire et à manger, puis elle lui dit qu'il aura une chambre à lui. Il hésite à accepter jusqu'à ce qu'il voie la jeune apparition au regard vert, ce qui le décide à rester après tout. Il demeure là, croit-il, environs six semaines dans un état de végétation virtuelle, et il passe son temps à observer les femmes discrètement (93). Alors, le regard de l'homme sur la Femme/les femmes est impliqué dans le sens du récit, comme nous avons déjà soupçonné en pensant aux mythes de la Méduse et d'Orphée ainsi que de la nouvelle de Colette. Le narrateur se sent suspendu, dans cette maison presque vide, à la fois entre ces deux femmes et tout autant qu'écarté par elles, sans parvenir à voir leurs visages, à part les yeux. Il croise de temps en temps le regard de la jeune dans de "brèves secondes d'humanité" à la fin de journées autrement "insensibles" (94), et il apprend que son nom est Emma.

Puis, un jour tout change. Dehors avant le lever du soleil et couché par terre, il se rend compte avec effroi qu'il ne sait plus qui il est ; il a perdu contact avec lui-même. Le temps passé avec les deux femmes mystérieuses semble ainsi lui avoir fait perdre son identité. En cherchant dans son portefeuille, il trouve la photographie d'une femme dont l'image floue ressemble assez à celle d'une femme en cagoule, peut-être même la tête de la jeune femme qui s'appelle Emma. En revenant vers la maison il voit, par la fenêtre de sa chambre, Emma complètement nue à part la cagoule en train de s'examiner dans la psyché du placard, le seul miroir de la maison. Il la regarde en train de se regarder. Il avait laissé la porte ouverte, contrairement aux ordres de la vieille lors de son arrivée. À ce moment-là, il s'était posé la question, s'agissait-il d'ordres, de conseils ou de prédictions (90). Alors il franchit la porte et la surprend, "fascinée par ce qu'elle y déchiffrait" (97). "L'énigme" s'habille et quitte la chambre. Il l'appelle par son nom, mais elle refuse le nom d'Emma disant que c'est un diminutif de Emmanuelle. Emmanuelle pourtant, nous dit le dictionnaire des noms, n'a rien à voir avec Emma. Emmanuelle est le féminin du nom qui veut dire, "Dieu en forme humaine"; Emma, comme nous le savons déjà, représente le tout, l'ensemble. Mais le corps et la tête de cette Emma ne sont pas réunis pour en faire un ensemble. Ils sont séparés par la cagoule. Donc, en refusant le nom d' "Emma" et en se réfugiant dans l'idéal du mystère de Dieu parmi nous, l'inconnaissable, en forme humaine, 
la jeune femme nous offre une image étrange-le corps sans tête désigné par un pseudonyme d'idéalisation et de mystère.

Par la suite, elle ne rencontre plus le regard du narrateur dans la salle à manger, plus de "brèves secondes d'humanité." Cette expérience du regard de l'homme sur le regard de la femme sur elle-même fait donc rupture dans le récit et sépare l'homme du mystère des yeux de la jeune femme, ce qu'il croit être son lien à l'humanité. Le regard de l'homme appréhendé chez la femme, bien qu'il ait porté seulement sur le corps, empêche le regard dans les yeux. Par contre, une nuit elle vient vers lui, ils font l'amour et, pendant qu'elle dort, il a envie de soulever sa cagoule, mais il se retient, ne sachant pas de quoi il a peur. Le corps de la femme, sans tête, fait de lui un objet idéal purement sexuel. Cet amour entre deux corps sans regard mutuel représente une partie de la vie, celle du concret, du matériel, de l'illusion impossible qui disparaît en fin de compte. L'absence du visage de la femme, bien que ses yeux soient en principe visibles, empêche pourtant la rencontre des esprits et des cœurs, ce qui est de l'abstrait, impossible à fixer, mais durable quand même. Le texte nous le dit: "J'ai compris que ses yeux étaient le temps qui passe et que son corps sans visage se confondait avec le monde" (97).

La nuit d'amour ne se répète pas et le narrateur décide alors qu'il faudrait partir, ce qu'il annonce aussitôt. La vieille lui demande de leur couper du bois pour l'hiver avant de partir, ce qu'il fait très volontiers. Du coup, la vieille amène la jeune Emma/Emman-uelle vers le narrateur en train de fendre un rondin. Qu'elle la tire par la main implique une accusation qui se prépare. La vieille a dû apprendre que la jeune avait couché avec l'homme, et lui avait permis de connaître son corps, sinon son cœur, et qu'il s'en va parce qu'elle ne couche plus avec lui. En relevant leurs deux cagoules, la vieille montre les visages mixtes qui révèlent ce que le narrateur voit comme l'horreur surpassant ses plus folles suppositions (102).

Le visage d'Emma était celui d'une femme de soixante-dix ans : la peau grisâtre, hachurée de profondes rides noires qui faisaient paraître ses yeux encore plus irréels, encore plus invraisemblablement lumineux. La vieille [. . . la vieille avait le visage jeune et régulier qu'aurait da avoir sa fille, si l'on exceptait les deux yeux glauques qui le souillaient comme deux crachats verdâtres sur une assiette de lait. (102-103).

Ces yeux de crachat effroyables l'attirent et il est fasciné par leur laideur : "J'eusse le sentiment qu'ils m'ordonnaient quelque chose ou plus 
précisément qu'ils cherchaient à réaliser à travers moi quelque impénétrable dessein" (103). Ce sont donc les ordres des yeux monstrueux de la vieille, selon le narrateur, qui sont responsables pour ce qui suit. Il se dit "abasourdi par cet inconcevable échange" (103) et, rempli d'effroi, d'angoisse, de douleur, il tranche brutalement les deux têtes d'un seul coup de hache.

Très méthodiquement, il prend la cuillère avec laquelle il avait remué son café au lait quelques heures plutôt, et il arrache délicatement les yeux d'Emma, les insérant dans la tête au visage jeune. Puis, il replace cette tête sur le corps jeune, reconstituant enfin l'image complète de la jeune et belle femme, dans une scène qui donne un nouveau sens au terme "chirurgie esthétique"! Il va chercher la photo de la cheminée qu'il avait vue le premier jour et il la compare à son Emma reconstituée, se disant: "Ces morceaux de chair assemblés à la hâte de la véritable Emmanuelle (je souligne), souriait en noir et blanc dans le creux de ma main" (104). Le corps a changé de nom : Le tout n'est pas un tout, mais une énigme, un idéal. Jetant un dernier coup d'œil sur le corps blanc et rouge, il prend la fuite et se retrouve couché par terre, sa femme sanglotant au-dessus de sa poitrine. Première pensée, c'est à cause de la photo d'Emma qu'il avait emportée... ce qui fait penser à Orphée essayant de sortir de l'Enfer avec Eurydice. Mais sur cette photo, constate-t-il avec stupeur et amertume, se trouve l'image d'un visage dissimulé sous une cagoule en forme de tâche brune, tâche de sang. Ce n'est ni l'ensemble d'Emma, ni l'énigme idéale d'Emmanuelle. En leur tranchant la tête, en insérant les yeux verts dans le visage à la peau tendue et aux traits réguliers, et ensuite en remettant la tête au corps jeune, il aurait tout gâché, il aurait détruit l'une et l'autre.

Revenons maintenant à nos premières idées, l'inter-texte des "Yeux d'Elsa" de Louis Aragon, puis les deux autres références, "La femme cachée" de Colette, et l'image Gestalt. Les Yeux d'Elsa forment un ensemble de poèmes qui expriment la dualité tragique de la deuxième guerre mondiale, de l'espoir soutenu par la résistance, qui se voient dans les yeux de la femme du poète. Les Yeux d'Elsa comprennent des "nuits" et des "plaintes." Les "nuits" font référence aux Nuits de mai d'Alfred de Musset, poète du dix-neuvième siècle, dont le thème est l'amour inassouvi. Et les "plaintes" nous ramènent encore plus loin dans le passé, au seizième siècle, même au Moyen Age, où elles sont la forme préférée pour un discours sur la discontinuité entre le passé et le présent (Adereth 17). Cela nous suggère le sens du titre de la nouvelle, la référence est très riche: Le nom Emma qui veut dire l'ensemble, le tout, la complétude, relié aux yeux est l'écho des "Yeux d'Elsa." Cela nous dit à la fois l'amour 
impossible ou incomplet, et la rupture entre le passé et le présent. Ce qui nous fait penser encore au titre de l'image en tête de notre texte, "Ma femme et ma belle-mère." Cet homme ne s'entend-il peut-être pas avec sa belle-mère? Ou bien, peut-être que la belle-mère lui montre-t-elle ce que deviendra sa femme avec le temps? Se lamente-t-il sur le passage du temps, la discontinuité entre l'idéal du passé, le corps beau et jeune de sa femme dans sa jeunesse, et ce qu'elle deviendra ou ce qu'elle est déjà devenue, le corps sans doute un peu fané?

Si nous nous souvenons que le mari de "La femme cachée," celui qui se dissimule sous une cagoule et en profite pour espionner sa femme en train de s'amuser seule découvrant ainsi qu'il ne la connaît pas et qu'il ne la connaîtra jamais, nous ne pouvons pas ignorer le côté énigmatique, le côté "Emmanuelle" de celle-ci. Alors, le mystère du texte se trouve dans l'écart, dans le mouvement, entre le connu et l'indéchiffrable, entre EMmA et Emmanuelle, entre les deux femmes, entre la jeunesse et la vieillesse, entre l'éternité et le temps qui fuit, entre l'imperfection et la perfection ou plutôt entre l'incomplet et le complet, car l'un des sens du mot parfait est justement complet. Ce n'est pas la différence des deux femmes, mais l'écart entre elles, l'herméneutique du mouvement entre les deux, qui permet leur appréhension.

Le sens du (ou d'un) texte se montre donc comme l'image qui surgit de l'arrière plan, qui se voit par rapport au champ arrière, c'est-à-dire en Relation de ce champ, pour reprendre le terme d'Édouard Glissant qui exprime une poétique qui "ne s'achève pas en absolu qualitatif" (47) mais qui est une totalité sans être totalitaire (148) ou encore "une synthèsegenèse jamais achevée" (188). Tel est le phénomène du rapport figure/ arrière-plan illustré dans l'image de la femme et la belle-mère. Tel est aussi le mystère de la perception, prise au dépourvu, peut-être sous forme de cauchemar de monstres. Peu importe ce qui se passe vraiment... il s'agit d'un récit de l'expérience intérieure du narrateur, de l'opportunité de prendre conscience des mystères de la vie, de la Femme, de sa femme, mais pas forcément de les déchiffrer, opportunité manquée. L'amertume qu'il éprouve au voir de la photo ensanglantée, apprend au lecteur qu'il n'a pas compris le sens de la dualité mixte, le sens en mouvement de l'énigme, il n'a pas trouvé le tout, l'ensemble, il n'a pas reconstitué Emma, et il n'a pas assez apprécié Emmanuelle.

Mais le lecteur prend conscience de ce que lui ne comprend pas. Le lecteur comprend ainsi qu'il n'y a pas d'Emma possible sans EMmanuelLe, mystère qui nous intrigue, qui nous fait nous questionner, qui nous fait poursuivre le chemin de la recherche de la compréhension. Le narrateur 
ne voit que l'idéal perdu d'une totalité unifiée, et ne comprend pas que cet idéal n'est qu'une partie de l'ensemble et non l'ensemble; l'idéal n'étant qu'un moyen de nous intéresser à la recherche d'un sens de la vie, mais totalement impossible de connaître. La totalité ouverte, l'ensemble, le parfait, le complet, EMMA n'est pas possible sans le processus, l'écart, la discontinuité, le mouvement, la Relation qu'implique(nt) EMMANUELLE (et sa mère) mystérieuse(s). Comme les deux femmes mystérieusement mélangées, le portrait des deux femmes sur une seule image, impossibles semble-t-il à faire surgir en même temps, implique le processus cognitif de l'esprit: la perception est un montage de ce que nous savons déjà sur ce que nous rencontrons, l'interprétation d'un récit est un processus de Relation incomplète et impossible à compléter. Ainsi ce mystère nous fait "connaître" l'inconnaissable peutêtre de la Femme ou de l'idéal, mais plus profondément, c'est le mystère de la compréhension humaine, la façon dont nous appréhendons notre monde-toujours en relation. Et, si nous décidons que c'est là où se trouve le vrai sens du récit, alors nous avons, contre toute attente, notre Emma, Emma qui est Emmanuelle. 


\section{Notes}

1. "Ma femme et ma belle-mère" Puck Magazine (1915) dans Richard J. Block \& Harold Yucker. Can You Believe Your Eyes (New York: Bruner Mazel, 1992).

2. Une autre version de ce texte a été présentée au colloque: Twentieth Century French Studies, University of California at Davis, le 30 mars 2001.

3. Mot allemand qui veut dire "forme" "ensemble" ou "configuration." Voir aussi le rapport de la figure/arrière-plan dans Hergenhahn 1992, 407-8.

4. Pour une discussion très claire des métaphores mortes, voir: George Lakoff et Mark Johnson, Metaphors We Live By, et Ruth Amossy, "Stéréotype et lecture," Stéréotypes et Clichés (Paris: Nathan, 1997) 7285. 


\section{Ouvrages cités}

Adereth, M. Aragon: The Resistance Poems, London: Grant \& Cutler, 1985.

Amossy, Ruth. Stéréotypes et Clichés, Paris: Nathan Université, 1997. Aragon, Louis. Les Yeux d'Elsa, Neuchâtel: Cahiers du Rhône, 1942. Block, Richard J. and Harold E. Yucker. Can You Believe Your Eyes? New York: Bruner/Mazel, 1992.

Chalvin, Antoine. "Les Yeux d'Emma." Les Yeux d'Emma, Paris: Alfil Editions, 1994.

Chevalier, Jean et Alian Gheerbrant. Dictionnaire des symboles, Paris: Robert Lafont, 1969.

Colette "La femme cachée." La femme cachée, Paris: Flammarion, 1924, 1951.

Glissant, Édouard. Poétique de la Relation, Paris: Gallimard, 1990.

Hergenhahn, B.R.An Introduction to the History of Psychology. Belmont, CA: Wads-worth, 1992.

Lakoff, George et Mark Johnson. Metaphors We Live By, Chicago: University of Chicago Press, 1980.

Musset, Alfred de. "Les Nuits de mai." CEuvres Complètes, Paris: Éditions de la Pléiade. 1963.

Samuelson, Pamela. Baby Names for the New Century, New York: Harper, 1994.

Wolman, Benjamin B. Dictionary of Behavioral Science, New York: Van Nostrand Reinhold, 1973. 
